\title{
Residents' Preferences and Performance of Three Techniques for Ultrasound-Guided Central Venous Cannulation After Simulation Training
}

\author{
Terrell Caffery, MD, ${ }^{1}$ Tonya Jagneaux, MD, ${ }^{2,3,4}$ Glenn N. Jones, PhD, ${ }^{5}$ Erik Stopa, DO, ${ }^{1}$ Nathan Freeman, MD, ${ }^{1}$ \\ Cara Cantelli Quin, MD, ${ }^{1}$ Ann C. Long, MD, MS, ${ }^{6}$ Lauren Zatarain, MD, ${ }^{2}$ Mandi W. Musso, PhD $^{1,7}$ \\ ${ }^{1}$ Emergency Medicine Residency Program, Louisiana State University Health Sciences Center School of Medicine, Baton Rouge, LA \\ ${ }^{2}$ Internal Medicine Residency Program, Louisiana State University Health Sciences Center School of Medicine, Baton Rouge, LA \\ ${ }^{3}$ Department of Quality and Safety, Our Lady of the Lake Regional Medical Center, Baton Rouge, LA ${ }^{4}$ Department of Internal Medicine, \\ Tulane University School of Medicine, New Orleans, LA ${ }^{5}$ Department of Family Medicine, Our Lady of the Lake Regional Medical Center, \\ Baton Rouge, LA ${ }^{6}$ Division of Pulmonary and Critical Care Medicine, Harborview Medical Center, University of Washington, Seattle, WA \\ ${ }^{7}$ Division of Academic Affairs, Our Lady of the Lake Regional Medical Center, Baton Rouge, LA
}

Background: Obtaining central venous cannulation of the internal jugular vein is an important skill for physicians to master. To our knowledge, no studies to date have examined residents' preferences or the safety of the oblique approach compared to other approaches. This study compared medical residents' preferences for and performance of ultrasound-guided central venous access using the transverse, longitudinal, and oblique approaches.

Methods: Emergency medicine and internal medicine residents $(n=72)$ at an urban community hospital participated in a central venous access course. To assess the residents' preferences, residents were asked to rank the transverse, longitudinal, and oblique approaches as first, second, or third. In addition to preference, skin-to-vein time, carotid artery puncture, and successful completion on the first attempt during a final skills analysis were measured.

Results: During the final skills analysis, the majority (87.5\%) of residents preferred the transverse approach. The oblique approach had a significantly larger proportion of failures of technique than the transverse approach $(P=0.02)$. No significant differences in successful cannulation on the first attempt, skin-to-vein time, or carotid artery puncture among the 3 approaches were found during the final skills assessment.

Conclusion: The majority of residents preferred the transverse approach to the longitudinal and oblique approaches. Although no significant differences among the 3 approaches were found in performance measures, more failures of technique occurred with the oblique approach. This study suggests that novices may require in-depth training and supervision to become proficient with the oblique approach.

Keywords: Central venous catheter, simulation training, ultrasonography

Address correspondence to Mandi W. Musso, PhD, Academic Research Director, Emergency Medicine Residency Program, Our Lady of the Lake Regional Medical Center, 5246 Brittany Drive, Baton Rouge, LA 70808. Tel: (225) 757-4148. Email: mmuss1@lsuhsc.edu

\section{INTRODUCTION}

Obtaining central venous access in the internal jugular vein is an important skill for physicians to master. The complication rate of this procedure is high; a 2015 review reported a complication rate of 135 per $1,000 .^{1}$ Complications of this procedure that can lead to potentially lethal complications include hemorrhage, hemothorax, pneumothorax, and carotid artery puncture. Before ultrasound was available, physicians performed this procedure essentially blindly using external anatomic landmarks. However, ultrasound technology has made it possible to visualize the vein during insertion of the needle, reducing line placement complications by $71 \%{ }^{1}$
Two traditional sonographic approaches used for central venous access are the transverse and longitudinal approaches. In the transverse (short-axis) approach, the internal jugular vein is imaged in the short axis with the needle inserted perpendicular to the sonographic plane. ${ }^{2}$ All structures can be visualized simultaneously during the procedure. However, the needle tip is not always visualized and may be difficult to follow, often resulting in posterior wall puncture of the internal jugular vein. ${ }^{3}$ The longitudinal (long-axis) approach allows users to visualize the internal jugular vein in the long axis and the needle tip during insertion. However, this approach does not promote visualization 
of other vessels or their relationship to the internal jugular. ${ }^{2} \mathrm{~A}$ newer technique, the oblique approach, optimizes imaging of the internal jugular vein and carotid artery and allows for visualization of the needle throughout the insertion of the catheter. ${ }^{4}$

To our knowledge, no studies to date have examined physician preferences or safety of the oblique technique compared to the other approaches. The purpose of this study was to examine residents' preferences for and performance of the transverse, longitudinal, and oblique approaches to central venous access after they received education and simulation training. We hypothesized that residents would prefer the oblique approach to the longitudinal and transverse approaches. We also hypothesized that the oblique approach would result in improved skin-to-vein time, a reduced number of attempts, and fewer carotid artery punctures compared to the longitudinal and transverse approaches.

\section{METHODS \\ Study Design}

This prospective study evaluated the effects of simulation training on the safety and utility of 3 ultrasound-guided central venous access approaches. The study was approved by the Louisiana State University School of Medicine Institutional Review Board. The project was funded by a Louisiana State University Health Sciences Center-New Orleans Academy for the Advancement of Educational Scholarship Grant.

\section{Study Setting and Population}

All emergency medicine and internal medicine residents at an urban community hospital participated in a central venous access course during the 2010-2011 academic year. Although training was mandatory, residents were given the option to have their data collected for research. One resident declined to participate. All other residents provided written, informed consent. The majority of residents recruited for this study reported some experience with central venous access. At the time of study recruitment, residents were overwhelmingly exposed to the transverse technique and, to a lesser extent, the longitudinal approach. Residents had not yet been exposed to the oblique technique because it had only recently been presented in the scientific literature.

\section{Education and Simulation Training}

Residents attended a lecture presented by ultrasoundtrained faculty members. They also watched a video on ultrasound-guided central venous access. ${ }^{5}$ Residents were given literature to review regarding the reduction in complications related to the use of ultrasound.$^{6-8}$ Residents also read an article on the oblique technique. ${ }^{4}$ Trained faculty members gave demonstrations of the technical aspects of each of the 3 approaches using a Blue Phantom simulation model (CAE Healthcare). This model includes both the internal jugular vein and carotid artery. A Sonosite M-Turbo (Fujifilm SonoSite, Inc.) with a high-frequency linear (HFL-25) transducer was used for ultrasound guidance. For the oblique technique, residents were instructed per the approach described by Phelan and Hagerty. ${ }^{4}$ After attending the lecture, residents participated in 90-minute skills sessions during which they were allowed to question and receive feedback from faculty members. The simulation training and assessment have been described in detail previously. ${ }^{9}$

\section{Measurement}

Resident performance was assessed during a final skills analysis. At that time, 2 faculty members or chief residents trained in observation and scoring performance of central venous catheter insertion observed and scored the residents. Percent agreement between the 2 raters was $>92 \%$ for all variables. Kappas ranged from 0.80 for number of attempts to 0.99 for both skin-to-vein time and type of approach. The raters were in $100 \%$ agreement for not-inplane attempts and for carotid artery puncture.

\section{Outcomes}

During the final skills analysis, residents were asked to choose the approach they felt most comfortable using. They were then asked to perform 2 additional attempts with their second and third choice approaches. Performance data included skin-to-vein time, carotid artery puncture, and successful completion on the first attempt. An attempt was defined as a single pass of the needle. Failure of technique was recorded when a resident chose not to attempt an approach, or the attempt did not truly reflect a particular approach.

\section{Statistical Analysis}

Descriptive statistics are provided for the skills analysis results. Skills assessment data that were lost or not interpretable were treated as missing. Failures of technique were treated as missing data for that attempt and omitted from analyses. Statistical significance was defined as $P \leq 0.05$. The rank order of preferences for the different approaches was treated as ordinal and analyzed using the Friedman test followed by the Wilcoxon signed-rank test as post hoc analysis. Logistic regression was used to explore whether experience, postgraduate year (PGY) level, or program influenced which technique the resident chose to attempt first. Binomial data, including failure of technique, successful cannulation on first attempt, and carotid puncture, were analyzed using the Cochran $Q$ test followed by the McNemar chi-square test. Skin-to-vein times (seconds) were analyzed with the repeated measures multivariate analysis of variance. The impact of PGY level on the binomial variables, such as carotid puncture, was analyzed using Fisher exact test. The impact of PGY level on skin-to-vein time was analyzed using repeated measures multivariate analysis of variance. All statistical testing was performed with SPSS v.23 (IBM Corp.).

\section{RESULTS}

Seventy-two residents gave consent for release of their performance data following their training. As detailed in Table 1, 37 were emergency medicine residents and 35 were internal medicine residents. Their experience with ultrasound-guided central venous cannulation varied considerably. All residents completed the training protocol.

During the final skills analysis, the majority (87.5\%) of residents preferred the transverse approach (Table 2). The transverse approach was preferred by significantly more residents $(P=0.01)$ compared to the oblique and the longitudinal approaches. Thirty-one of the 37 emergency medicine residents $(83.8 \%)$ and 32 of the 35 internal medicine residents $(91.4 \%)$ chose the transverse approach first, suggesting no difference $(P>0.48)$ between the programs. No 
Table 1. Residents' Previous Experience with Ultrasound-Guided Central Venous Cannulation (US-CVC)

\begin{tabular}{|c|c|c|c|c|}
\hline Residents & $\mathbf{n}$ & $\begin{array}{c}\text { US-CVC } \\
\text { Observed }\end{array}$ & $\begin{array}{c}\text { US-CVC } \\
\text { Completed } \\
\text { With Assistance }\end{array}$ & $\begin{array}{c}\text { US-CVC } \\
\text { Completed } \\
\text { Without Assistance }\end{array}$ \\
\hline Emergency Medicine & 37 & & & \\
\hline PGY 1 & 14 & $7.6(4.2)$ & $4.5(5.0)$ & $0.6(1.5)$ \\
\hline PGY 2 & 14 & $23.8(32.1)$ & $15.2(23.0)$ & $15.8(17.7)$ \\
\hline PGY 3 & 9 & $41.1(60.3)$ & $13.5(10.9)$ & $51.7(23.7)$ \\
\hline Internal Medicine & 35 & & & \\
\hline PGY 1 & 16 & $5.9(10.9)$ & $2.5(2.0)$ & $0.0(0.0)$ \\
\hline PGY 2 & 8 & $11.2(7.4)$ & $11.9(18.8)$ & $5.6(10.2)$ \\
\hline PGY 3 & 11 & $11.6(12.7)$ & $11.5(8.6)$ & $15.5(8.2)$ \\
\hline
\end{tabular}

Data are presented as the mean (SD) number of US-CVCs.

PGY, postgraduate year.

difference in preferences based on PGY level was detected $(P=1.00)$, as $86.7 \%$ of PGY $1,86.4 \%$ of PGY 2 , and $90.0 \%$ of PGY 3 residents chose the transverse approach first. None of the 3 indices of experience presented in Table 1 was predictive of which approach was chosen first $(P=0.85)$. Although $12.5 \%$ of the residents chose the oblique approach as their preferred approach and none chose longitudinal, the difference in ranking was not statistically significant $(P>0.06)$. Overall, the transverse approach was the favored method, followed by the oblique and longitudinal approaches.

A significantly larger proportion of failures of technique occurred with the oblique approach than the transverse approach $(P=0.02)$. One resident failed to find the correct ultrasound settings and in essence failed every approach attempted. All residents attempted the transverse technique. One resident chose not to attempt the longitudinal technique. Six residents had problems executing the oblique technique. Two residents repeated the transverse or longitudinal approaches rather than attempt the oblique approach. Four residents attempted an oblique approach, but the needle was not in plane, meaning that they took a hybridized approach, using the oblique ultrasound view but the trans- verse needle approach. One of these residents punctured the carotid artery.

No significant differences were seen in successful cannulation on the first attempt, skin-to-vein times, or carotid artery punctures among the 3 approaches during the final skills assessment. Table 3 presents the results data by PGY level. The only significant difference was in successful cannulation on first attempt using the transverse approach; the interns were less successful than the PGY 2 and PGY 3 residents $(P=0.05)$.

\section{DISCUSSION}

This study resulted in 3 noteworthy findings. First, the residents in our cohort overwhelmingly favored the transverse approach. Second, the oblique approach appears to be more technically difficult to master compared to the other 2 approaches, given the significantly higher number of failures with this approach. Finally, no significant differences were seen in the number of attempts to successful completion, skin-to-vein times, or carotid punctures among the 3 approaches.

Data on the preference for and safety of the longitudinal vs transverse approaches are limited and mixed. Blaivas and

Table 2. Resident Performance During the Final Skills Assessment

\begin{tabular}{|c|c|c|c|c|}
\hline Variable & $\begin{array}{l}\text { Transverse } \\
\text { Approach }\end{array}$ & $\begin{array}{l}\text { Oblique } \\
\text { Approach }\end{array}$ & $\begin{array}{l}\text { Longitudinal } \\
\text { Approach }\end{array}$ & $P$ Value \\
\hline $\begin{array}{l}\text { Residents' preferred approach } \\
\text { to central venous access, } \%(95 \% \mathrm{Cl})\end{array}$ & & & & 0.01 \\
\hline First choice & $87.5^{\mathrm{a}}(78.4-93.6)$ & $12.5^{\mathrm{b}}(6.4-21.6)$ & $0.0^{\mathrm{b}}(0.0-3.4)$ & \\
\hline Second choice & $9.7^{\mathrm{a}}(4.5-18.1)$ & $44.4^{\mathrm{b}}(33.4-56.0)$ & $45.8^{\mathrm{b}}(34.7-57.3)$ & \\
\hline Third choice & $2.8^{\mathrm{a}}(0.6-8.6)$ & $43.1^{\mathrm{b}}(32.1-54.6)$ & $54.2^{\mathrm{b}}(42.7-65.3)$ & \\
\hline Failure of technique, \% (95\% Cl) & $1.4^{\mathrm{a}}(0.2-6.3)$ & $9.7^{\mathrm{b}}(4.5-18.1)$ & $2.8^{\mathrm{ab}}(0.6-8.6)$ & 0.02 \\
\hline Successful cannulation on first attempt, \% (95\% Cl) & $78.9(68.3-87.1)$ & $76.9(65.7-85.9)$ & $82.9(72.8-90.3)$ & ns \\
\hline Carotid artery puncture, $\%(95 \% \mathrm{Cl})$ & $0.0(0.0-3.5)$ & $1.5(0.2-7.0)$ & $7.1(2.8-14.9)$ & ns \\
\hline Skin-to-vein time, seconds, mean $(95 \% \mathrm{Cl})$ & $17.6(12.1-23.2)$ & $19.2(13.4-25.0)$ & $18.4(12.3-24.4)$ & ns \\
\hline
\end{tabular}

\footnotetext{
${ }^{a, b}$ For each variable, means or proportions in a line with the same or no superscript do not differ $(P>0.05)$. Statistical significance is defined as $P \leq 0.05$. $\mathrm{Cl}$, confidence interval; ns, nonsignificant.
} 
Table 3. Resident Performance During the Final Skills Assessment by Postgraduate Year (PGY) Level

\begin{tabular}{|c|c|c|c|}
\hline Variable & Transverse Approach & Oblique Approach & Longitudinal Approach \\
\hline \multicolumn{4}{|c|}{$\begin{array}{l}\text { Residents' preferred approach } \\
\text { to central venous access, } \%(95 \% \mathrm{Cl})\end{array}$} \\
\hline PGY 1 & $86.7(71.3-95.3)$ & $13.3(4.7-28.7)$ & $0.0(0.0-0.8)$ \\
\hline PGY 2 & $86.4(67.9-96.0)$ & $13.6(4.0-32.1)$ & $0.0(0.0-10.7)$ \\
\hline PGY 3 & $90.0(71.6-97.9)$ & $10.0(2.1-28.4)$ & $0.0(0.0-11.7)$ \\
\hline \multicolumn{4}{|c|}{ Failure of technique, $\%(95 \% \mathrm{Cl})$} \\
\hline PGY 1 & $3.3(0.4-14.5)$ & $6.7(1.4-19.7)$ & $6.7(1.4-19.7)$ \\
\hline PGY 2 & $0.0(0.0-10.7)$ & $18.2(6.5-37.6)$ & $0.0(0.0-10.7)$ \\
\hline PGY 3 & $0.0(0.0-11.7)$ & $5.0(0.5-21.1)$ & $0.0(0.0-11.7)$ \\
\hline \multicolumn{4}{|c|}{ Successful cannulation on first attempt, \% (95\% Cl) } \\
\hline PGY 1 & $62.1(44.0-77.9)^{a}$ & $67.9(49.5-82.8)$ & $71.4(53.2-85.5)$ \\
\hline PGY 2 & $90.9(73.9-98.1)^{\mathrm{b}}$ & $83.3(61.9-95.1)$ & $90.9(73.9-98.1)$ \\
\hline PGY 3 & $90.0(71.6-97.9)^{\mathrm{b}}$ & $84.2(63.6-95.3)$ & $90.0(71.6-97.9)$ \\
\hline \multicolumn{4}{|c|}{ Carotid artery puncture, $\%(95 \% \mathrm{Cl})$} \\
\hline PGY 1 & $0.0(0.0-8.2)$ & $3.6(0.4-15.5)$ & $10.7(3.1-25.9)$ \\
\hline PGY 2 & $0.0(0.0-10.7)$ & $0.0(0.0-12.9)$ & $4.5(0.5-19.3)$ \\
\hline PGY 3 & $0.0(0.0-11.7)$ & $0.0(0.0-12.2)$ & $5.0(0.5-21.1)$ \\
\hline \multicolumn{4}{|c|}{ Skin-to-vein time, seconds, mean $(95 \% \mathrm{Cl})$} \\
\hline PGY 1 & $23.2(13.9-32.6)$ & $24.5(16.5-32.5)$ & $25.5(15.6-35.5)$ \\
\hline PGY 2 & $15.1(3.7-26.5)$ & $11.9(2.1-21.7)$ & $11.7(-0.5-23.9)$ \\
\hline PGY 3 & $13.0(1.9-24.1)$ & $14.3(4.8-23.8)$ & $18.1(6.2-30.0)$ \\
\hline
\end{tabular}

\footnotetext{
${ }^{\mathrm{a}, \mathrm{b}}$ For each variable, means or proportions in the same column with the same or no superscript do not differ $(P>0.05)$. Statistical significance is defined as $P \leq 0.05$.

$\mathrm{Cl}$, confidence interval.
}

colleagues reported that novice ultrasound users were able to gain access more quickly using the transverse approach than the longitudinal approach. ${ }^{10}$ In their study, skin breaks, needle redirections, and difficulty scores were not different between the 2 approaches. Vogel and colleagues reported that among emergency medicine residents, the long-axis view was more efficient than the short-axis view for internal jugular central venous access. ${ }^{11}$ Phelan and Hagerty anecdotally noted that residents and interested staff who have learned the oblique technique report it is easier to perform and optimizes needle visualization. ${ }^{4}$ Conversely, in our study, residents preferred the transverse technique after training. A noteworthy point is that our residents had a wide range of experience with central venous access, but all of them had primarily been exposed to the transverse approach prior to the study. However, our subsequent analyses suggest prior experience was not related to preference or most measures of performance.

The results of this study indicate that teaching the oblique approach may require more training than one lecture and several simulation attempts, as a significantly larger proportion of residents failed the oblique technique compared to the other 2 techniques. The failure rate may be related to the residents' lack of familiarity with the oblique approach. In our study, however, we found no significant differences in number of attempts, skin-to-vein times, or carotid punctures among the 3 approaches when the oblique technique was performed correctly. In the years since this study was conducted, residents in our emergency medicine and internal medicine residency programs have received yearly lectures that include information about transverse, longitudinal, and oblique techniques, including the benefits and risks of each procedure. Additionally, residents are taught to use all 3 techniques in a simulation laboratory, and they are required to practice each technique with regular frequency. Anecdotally, this practice has resulted in residents becoming far more proficient in using all 3 techniques.

The literature suggests that with in-depth ultrasound training and experience, residents may find that the oblique technique has significant advantages for obtaining central venous access. A randomized crossover study reported significantly less overlap between the internal jugular vein and carotid artery using an oblique view compared to a transverse view; however, this study did not assess clinical outcomes of various approaches to central venous access. ${ }^{12}$ Wilson and colleagues reported no statistically significant differences in posterior vessel wall puncture, number of attempts, or skin-to-vein time in residents and attendings using the oblique approach compared to the transverse approach in simulated models. ${ }^{13}$ However, participants reported greater confidence in the needle tip being located in the lumen of the vein when using the oblique approach.

Limitations of this study include a limited sample of residents from one hospital that may limit the generalizability 
of this study. Further studies should examine the utility and safety of the oblique approach in multiinstitution settings and among experienced providers. We did not measure how frequently residents had been exposed to the transverse vs longitudinal techniques prior to this study, although anecdotally, the transverse approach was used by the majority of residents prior to the study. However, not having this data limits our ability to examine the effect on preferences of prior exposure to these techniques. Our study was conducted during the 2010-2011 academic year. In 2012, Dilisio and Mittnacht described a modification of the oblique technique called the medial-oblique approach that further minimizes the risk of carotid puncture. ${ }^{14}$ Additional research is needed to investigate whether this modification affects residents' preferences for approach and their performance in obtaining central venous access. We did not survey residents regarding their perceptions, including perceived difficulty, of the 3 approaches. Such data would have yielded information that could help tailor future training courses.

\section{CONCLUSION}

Despite the oblique approach's advantage of optimizing the view of internal vessels and the needle tip during central venous line placement, the vast majority of residents in our cohort selected the transverse approach as their first choice. A trend in the data suggested that the residents preferred the oblique approach to the longitudinal approach. We found no significant differences in most performance measures among the 3 approaches, but more failures of technique occurred with the oblique approach. This study suggests that research is needed to develop comprehensive simulation protocols that allow novices to become comfortable and proficient with the oblique approach.

\section{ACKNOWLEDGMENTS}

Dr Terrell Caffery and Dr Tonya Jagneaux received a Louisiana State University Health Sciences Center-New Orleans Academy for the Advancement of Educational Scholarship Grant to fund this project.

This project was made possible by the volunteer efforts of the following individuals: Michelle Bordelon, research coordinator data entry/organization; Darren Barefield, MD; Stephen Brierre, MD; Roy Culotta, MD; John Godke, MD; Hollis O'Neal, MD; Kevin Reed, MD; Chris Thomas, MD, Louisiana State University Health Sciences Center Baton Rouge faculty-education/evaluation.

\section{REFERENCES}

1. Brass P, Hellmich M, Kolodziej L, Schick G, Smith AF. Ultrasound guidance versus anatomical landmarks for subclavian or femoral vein catheterization. Cochrane Database Syst Rev. 2015 Jan 9;1:CD011447. doi: 10.1002/14651858.CD011447.
2. Weiner MM, Geldard P, Mittnacht AJ. Ultrasound-guided vascular access: a comprehensive review. J Cardiothorac Vasc Anesth. 2013 Apr;27(2):345-360. doi: 10.1053/j.jvca.2012.07.007.

3. Blaivas M, Adhikari S. An unseen danger: frequency of posterior vessel wall penetration by needles during attempts to place internal jugular vein central catheters using ultrasound guidance. Crit Care Med. 2009 Aug;37(8):2345-2349; quiz 2359. doi: 10.1097/CCM.0b013e3181a067d4.

4. Phelan M, Hagerty D. The oblique view: an alternative approach for ultrasound-guided central line placement. J Emerg Med. 2009 Nov;37(4):403-408. doi: 10.1016/j. jemermed.2008.02.061.

5. Graham AS, Ozment C, Tegtmeyer K, Lai S, Braner DA. Videos in clinical medicine. Central venous catheterization. $N$ Engl J Med. 2007 May 24;356(21):e21.

6. Karakitsos D, Labropoulos N, De Groot E, et al. Real-time ultrasound-guided catheterisation of the internal jugular vein: a prospective comparison with the landmark technique in critical care patients. Crit Care. 2006;10(6):R162.

7. Pronovost $P$, Needham $D$, Berenholtz $S$, et al. An intervention to decrease catheter-related bloodstream infections in the ICU. N Engl J Med. 2006 Dec 28;355(26):2725-2732.

8. Ortega R, Song M, Hansen CJ, Barash P. Videos in clinical medicine. Ultrasound-guided internal jugular vein cannulation. N Engl J Med. 2010 Apr 22;362(16):e57. doi: 10.1056/ NEJMvcm0810156.

9. Jagneaux T, Caffery T, Musso MW, et al. Simulation-based education enhances patient safety behaviors during central venous catheter placement. J Patient Saf. 2017 Oct 4. [Epub ahead of print]. doi: 10.1097/PTS.0000000000000425.

10. Blaivas M, Brannam L, Fernandez E. Short-axis versus long-axis approaches for teaching ultrasound-guided vascular access on a new inanimate model. Acad Emerg Med. 2003 Dec;10 (12):1307-1311.

11. Vogel JA, Haukoos JS, Erickson CL, et al. Is long-axis view superior to short-axis view in ultrasound-guided central venous catheterization? Crit Care Med. 2015 Apr;43(4):832-839. doi: 10.1097/CCM.0000000000000823.

12. Baidya DK, Chandralekha, Darlong V, Pandey R, Goswami D, Maitra S. Comparative sonoanatomy of classic "short axis" probe position with a novel "medial-oblique" probe position for ultrasound-guided internal jugular vein cannulation: a crossover study. J Emerg Med. 2015 May;48(5):590-596. doi: 10.1016/j.jemermed.2014.07.062.

13. Wilson JG, Berona KM, Stein JC, Wang R. Oblique-axis vs. short-axis view in ultrasound-guided central venous catheterization. J Emerg Med. 2014 Jul;47(1):45-50. doi: 10.1016/ j.jemermed.2013.11.080.

14. Dilisio R, Mittnacht AJ. The "medial-oblique" approach to ultrasound-guided central venous cannulation-maximize the view, minimize the risk. J Cardiothorac Vasc Anesth. 2012 Dec;26(6):982-984. doi: 10.1053/j.jvca.2012.04.013.

This article meets the Accreditation Council for Graduate Medical Education and the American Board of Medical Specialties Maintenance of Certification competencies for Patient Care, Medical Knowledge, and Practice-Based Learning and Improvement. 